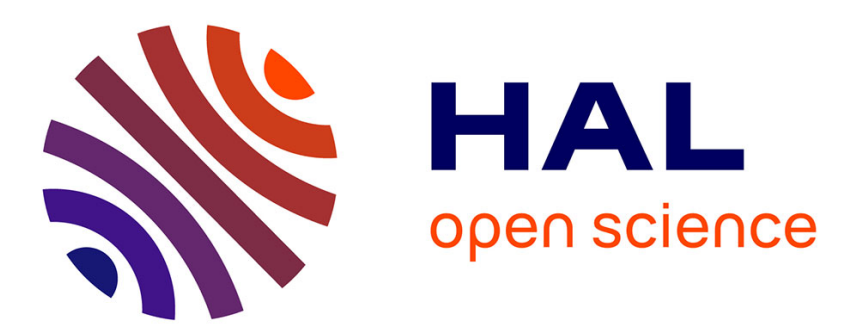

\title{
Transitions urbaines des anciennes villes de garnison en Allemagne: l'exemple de Landau in der Pfalz
}

\author{
Denis Mathis, Anne Mathis
}

\section{To cite this version:}

Denis Mathis, Anne Mathis. Transitions urbaines des anciennes villes de garnison en Allemagne: l'exemple de Landau in der Pfalz. Revue Géographique de l'Est, 2017, 57 (3-4), 10.4000/rge.7526 . hal-02813234

\section{HAL Id: hal-02813234 \\ https://hal.science/hal-02813234}

Submitted on 6 Jun 2020

HAL is a multi-disciplinary open access archive for the deposit and dissemination of scientific research documents, whether they are published or not. The documents may come from teaching and research institutions in France or abroad, or from public or private research centers.
L'archive ouverte pluridisciplinaire HAL, est destinée au dépôt et à la diffusion de documents scientifiques de niveau recherche, publiés ou non, émanant des établissements d'enseignement et de recherche français ou étrangers, des laboratoires publics ou privés. 


\section{Transitions urbaines des anciennes villes de garnison en Allemagne : l'exemple de Landau in der Pfalz}

Urban transitions of the ancient garrison towns in Germany: the example of Landau in der Pfalz

Städtischen Übergänge der Städtegarnison in Deutschland: das Beispiel von

Landau in der Pfalz

Denis Mathis et Anne Mathis

\section{OpenEdition}

Journals

Édition électronique

URL : http://journals.openedition.org/rge/7526

ISSN : 2108-6478

Éditeur

Association des géographes de l'Est

Référence électronique

Denis Mathis et Anne Mathis, «Transitions urbaines des anciennes villes de garnison en Allemagne: l'exemple de Landau in der Pfalz », Revue Géographique de l'Est [En ligne], vol. 57 / 3-4 | 2017, mis en ligne le 12 avril 2019, consulté le 14 novembre 2019. URL : http://journals.openedition.org/rge/7526

Ce document a été généré automatiquement le 14 novembre 2019.

Tous droits réservés 


\title{
Transitions urbaines des anciennes villes de garnison en Allemagne : l'exemple de Landau in der Pfalz
}

\author{
Urban transitions of the ancient garrison towns in Germany: the example of \\ Landau in der Pfalz \\ Städtischen Übergänge der Städtegarnison in Deutschland: das Beispiel von \\ Landau in der Pfalz
}

Denis Mathis et Anne Mathis

1 Les transitions géopolitiques de la période post-Guerre froide ont eu d'importantes conséquences sur l'organisation des territoires de défense en modifiant les logiques et l'organisation des déploiements militaires. Comme l'affirmait le Livre Blanc de la défense de 1994, "la France se trouve dans la situation peu familière, où ses frontières ne semblent plus immédiatement et directement menacées ${ }^{1}$. Aussi, les hypothèses stratégiques et les logiques de déploiements qui avaient prévalu depuis la Guerre froide notamment par la présence des Forces Françaises en Allemagne (F.F.A.) ont été progressivement remises en cause conjointement avec la loi de professionnalisation des armées et l'adaptation aux risques géostratégiques. Ce processus de rééquilibrages territoriaux et d'ajustement des moyens militaires, non propre à la France, a entraîné dans toute l'Europe un déménagement du territoire (Subra, 2018) avec comme conséquences les enjeux de requalification des friches urbaines et des périmètres militaires abandonnés. L'ampleur du phénomène a pu se mesurer à Metz dont le déclin de la fonction militaire s'est opéré après une large diminution des effectifs (Mathis et Mathis, 2015). Le maillage structurant des déploiements militaires a été lentement recomposé de 1995 à nos jours et la question du retraitement des friches militaires constitue un enjeu majeur pour de nombreuses villes de garnison, désormais partiellement ou totalement démilitarisées (Mathis, 2016a ; Mathis, 2011). L'approche géohistorique des processus de démilitarisation permet d'observer les étapes de la transition fonctionnelle et de recomposition des villes de garnison désormais déclassées. À l'échelle des territoires urbains, ce processus de déménagement est subi et imposé brutalement. Il constitue 
une rupture dans une histoire et un récit urbain de la militarisation associé à un contexte géopolitique.

Par transition, il faut comprendre le processus de transformation/mutation, tant fonctionnelle que paysagère, qui s'opère au sein de l'ancienne ville citadelle ou de l'ancienne ville de garnison de Landau. La transition peut se résumer par la phase intermédiaire entre deux états de l'urbain : la ville militaire et la ville post-militaire reprenant en cela la définition notionnelle de B. Elissalde (2000) pour qui la «transition territoriale permet de comprendre par exemple comment la domination d'un système productif s'ajuste plus ou moins vite aux systèmes spatiaux antérieurs et produit des configurations territoriales différenciées». Cependant, nous retiendrons la transition dans une dimension chronologique plus globale, c'est-à-dire comme le processus de transformation de l'espace urbain allant de la situation antérieure à la situation posttransition. De fait, il nous paraît nécessaire d'analyser ce processus sans rompre le long linéament d'une histoire et d'un récit urbain : c'est le temps du paysage géographique qui incarne un fragment d'histoire solidifiée. À l'échelle temporelle des phases de régénération urbaine, il s'agit d'un temps cyclique, mais aussi d'un temps ressenti (George, 1967). De cette façon, il est question de souligner le processus d'adaptation de la ville et d'amorcer une lecture prospective de celle-ci. Afin d'observer et d'analyser les processus des transitions urbaines à Landau, nous retiendrons comme entrée principale une approche paysagère et géohistorique de l'héritage militaire. Cette dernière doit servir de support afin de comprendre comment une ancienne ville militarisée parvient par des jeux de patrimonialisation et des expériences urbaines innovantes à construire un projet de conversion destiné à gommer les effets sociodémographiques du départ de l'armée. À Landau, le paysage de la militarisation est par ses aspects cumulatifs, le paysage urbain historique dont la conservation, la protection ou la reconstruction sont un enjeu culturel et identitaire. Malgré la déconnexion entre paysage et fonction liée à la démilitarisation, l'héritage militaire sert encore de support au processus de fabrique de la ville.

3 Concernant Landau, la question de l'héritage militaire pourrait apparaître difficile à gérer car comme souvent dans les régions frontalières structurées en territoire de Défense, les legs des déploiements militaires sont multiformes et hybridés. Ils traduisent les fluctuations des frontières et des souverainetés. Cette question des " frontières fantômes $~ "^{2}$ faites d'héritages culturels est clairement assumée. L'exemple de Landau illustre une démarche où la question de la démilitarisation n'est pas considérée comme une catastrophe, même si elle rompt avec une organisation fonctionnelle de la ville. Elle est une opportunité qui permet de construire une transition fonctionnelle sans rupture ni discontinuité du récit urbain construit autour de l'empreinte militaire. La valorisation de cet héritage permet de soutenir un discours et une démarche opératoire destinée à préparer les transformations de «la ville de demain $»^{3}$. L'exploitation de cette mémoire militaire sert de cadre aux aménagements territoriaux à échelle de la ville ou d'un territoire (Ginet et Wiesztort, 2013). L'approche géhistorique permet de souligner que ce processus a déjà été effectué à la fin du XIX ${ }^{\mathrm{e}}$ siècle avec la transformation de la ville-citadelle en ville de garnison. La démarche de reconversion des espaces militaires réalisée alors avait transformé la ville, l'adaptant aux logiques nouvelles de l'organisation des transports et aux mobilités. La nouvelle phase de démilitarisation qui s'opère avec le départ progressif des F.F.A. permet, dans un premier temps, dans le contexte de la charte d'Aalborg (1994), d'expérimenter la 
mise en œuvre d'écoquartier sur l'ancien quartier militaire Mangin devenu l'écoquartier Vauban, puis dans un second temps de planifier la "ville de demain " pour les espaces péricentraux, en disposant notamment de l'imposante emprise du quartier Foch-Estienne. Paradoxalement, le retraitement des espaces démilitarisés ne s'est pas opéré comme une amputation de l'urbain suivant une logique de recompactage et de décroissance, mais plutôt suivant une logique de réappropriation de ces lieux au sein de l'espace urbain. Il s'agit d'en faire un atout, dans un dessein de relance de l'écosystème urbain et d'assurer la transformation de la ville. Pour souligner ces transitions urbaines, nous présenterons dans un premier temps le processus ancien de la transition entre la ville-citadelle et la ville de garnison, puis dans un second temps la seconde transition entre la ville militaire et la ville post-militaire à l'échelle des anciens quartiers et enfin dans un troisième temps les formes de la régénération à l'échelle de la ville.

\section{La transition de l'urbanisme vaubanien à la ville de garnison (XVIIIe s.-2009)}

4 Landau in der Pfalz (ou Landau en Palatinat en français) est une petite ville allemande (46 $000^{4}$ en ce qui concerne une kreisfreie Stadt ${ }^{5}$, créée en 1972). La population du quartier de centre-ville (Landau) comptait 31000 habitants en 2011 Son destin chahuté entre la France et l'Allemagne a profondément marqué les paysages urbains en laissant en héritage les traces et marques de la militarisation qu'elle soit française ou allemande (fig.1). Son long passé militaire débuté sous le règne de Louis XIV s'est achevé avec le départ des dernières unités françaises en 2009. Ville guerrière, puis ville de garnison, le destin de Landau fut lié à l'armée qui aujourd'hui a déserté la ville.

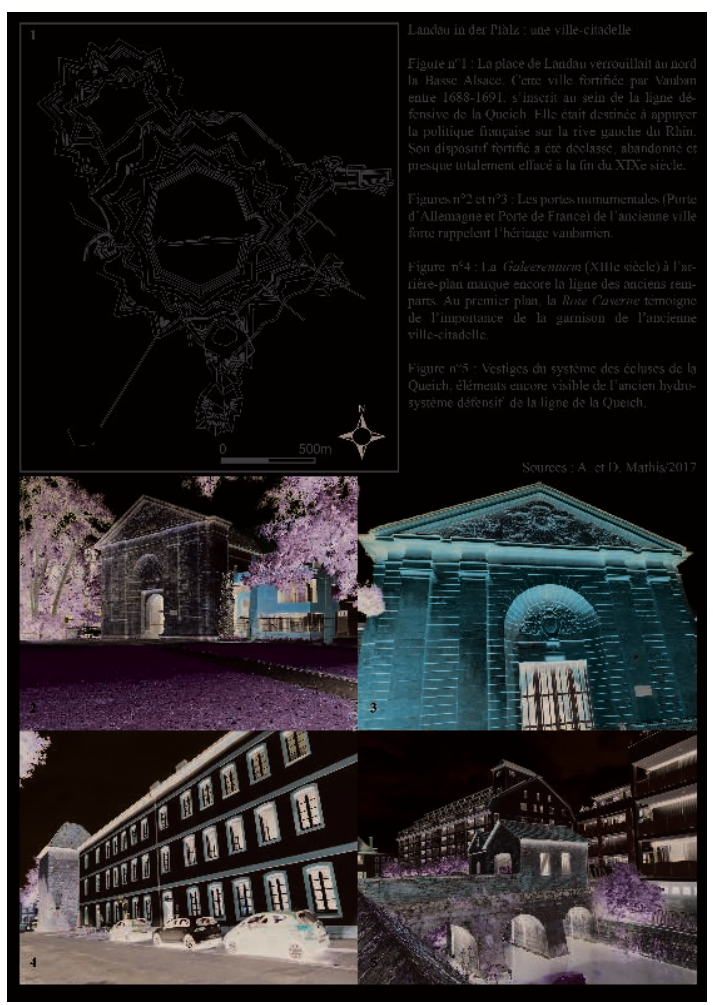




\section{La production de la ville-citadelle}

5 Appréhender sur un «temps long » les processus de production des villes de garnison permet de mettre en évidence les étapes de transitions des espaces urbains. La militarisation urbaine dans les processus de fabrique de la ville montre une contrainte majeure où la fonction impose ses ruptures et ses discontinuités. La démilitarisation, qu'elle soit totale ou partielle, offre des opportunités en levant progressivement les contraintes. Elle rend alors possibles les transformations urbaines.

6 À Landau, la première phase de militarisation a débuté avec Sébastien de Presles de Vauban (1633-1707) (fig.2 et 3). La vieille cité médiévale entre dans une phase de déconstruction-reconstruction propre au processus de conquête territoriale et à la mise en défense des nouveaux territoires. Ainsi, les anciens remparts médiévaux ont été effacés pour laisser la place à une ville-citadelle moderne (1688-1691). Seule la Galeerenturm témoigne encore de l'ancien système fortifié (fig. 4). Pour les besoins de la construction, le canal d'Alberweiler est creusé à l'ouest de la ville pour permettre l'acheminement des pierres de bonne qualité. Par la suite, la Queich a été aménagée pour structurer l'espace de défense. La ville pouvait ainsi être isolée par un système d'inondation commandé par des maisons éclusières (fig.5), puis par la mise en œuvre de la ligne défensive de la Queich établie du massif du Pfälzerwald au Rhin et organisant un hydrosystème de défense (Mathis, 2016b). Ce territoire de défense traduit dans l'espace la mise en œuvre des nouvelles frontières militaires. Ces dernières doivent " s'articuler sur de solides bases car elle défend le royaume et sert de base à des opérations militaires » (L. Jalabert 2011).

7 Landau devient une ville-citadelle inscrite dans un important système bastionné, renforcé par un fort couvrant au nord la porte d'Allemagne. Lieu de concentration des forces militaires, point d'appui et pivot pour des campagnes en Allemagne, la ville accueille au cœur de ses murs : 5 casernes dont les constructions s'échelonnent de 1692 à $1759^{6}$, un hôpital de guerre de 1732 à 1739 , deux magasins à poudre et un bâtiment du siège construits en 1742 .

\section{Le déclassement et la transformation en ville de garnison}

8 Perdue par la France en 1815, la forteresse de Landau devient forteresse fédérale en 1816 dans le cadre de la Confédération germanique (1815-1866) et reçoit une garnison bavaroise. Deux nouvelles casernes sont construites ou rénovées: la nouvelle Reiterkaserne (1818-1819) et surtout le Fort Barrack (1861-1863). Landau est devenu une cité guerrière ainsi qu'une ville de garnison. Cependant, la recomposition géopolitique et territoriale à la fin de l'empire français occasionne le développement à quelques kilomètres de la place prussienne de Germersheim. Enfin, l'arrivée du chemin de fer entraîne le percement des remparts et la construction de la gare en 1855. Un quartier industriel se développe alors. Landau entre dans un processus de démilitarisation des fortifications annonçant ce que Rocolle (2002) nomme la fin des cités guerrières. Toutefois, c'est la guerre franco-prussienne de 1870-1871 et l'annexion de l'Alsace et de la Moselle (1871-1918) qui bouleversent la donne géostratégique du Palatinat. En effet, ce territoire n'est plus directement exposé à une invasion française. La place de Gemersheim conserve une fonction défensive car intégrée dans le système du Rhin alors que Landau n'a plus de valeur stratégique. Dans le contexte de 
reconfiguration du territoire de défense du nouvel empire allemand, la place forte entre dans un processus de démantèlement qui débute en 1872 et s'effectue en deux temps, de 1871 à 1895 puis de 1919 à 1921, en application du traité de Versailles.

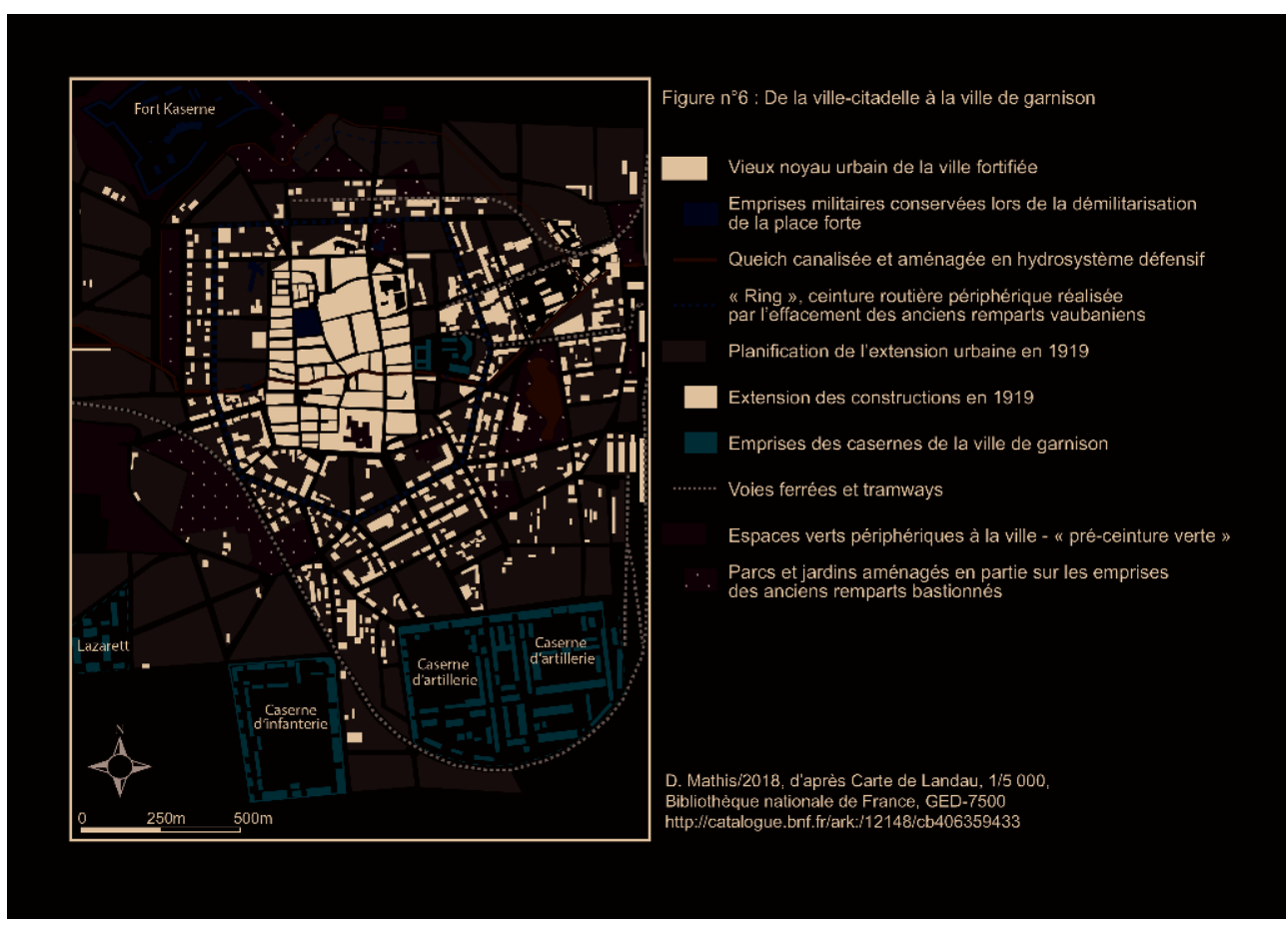

9 Cependant, le démantèlement des remparts n'entraîne pas de processus de démilitarisation. Le renforcement des frontières militaires entraîne une dilatation des villes de garnison qui déploient sur leurs périphéries de grands casernements reliés au réseau ferroviaire (fig.6). En même temps certains casernements internes à la ville sont détruits car l'espace urbain est jugé trop exigu. Ainsi, la fin des fonctions défensives n'a pas entraîné le déclassement de la ville. Le renforcement de la garnison offre même un nouvel essor urbain. L'effacement des remparts permet de créer une réserve foncière de $280000 \mathrm{~m}^{2}$. Certaines vieilles casernes urbaines au cœur de la ville sont abandonnées, renforçant les disponibilités foncières : la démilitarisation reste alors une opportunité foncière (Mathis et Mathis, 2015).

10 Seul l'espace du fort échappe aux travaux de démolition. Les portes monumentales sont conservées, tel un décor urbain, désormais remis en scène comme un marqueur des traces de l'empreinte vaubanienne (fig.2 et 3). Parallèlement, il faut refaire la ville, c'est-à-dire profiter de la levée des contraintes liées au déclassement de la forteresse. Il s'agissait déjà à l'époque d'une opportunité dans le processus de fabrique de la ville. En effet quand tant de villes-forteresses en Europe sont restées contraintes, inscrites et cernées par l'ancienne enceinte périphérique des remparts malgré la levée des restrictions militaires, Landau a réussi à saisir l'opportunité de refaçonner et de lier centre et périphéries par la mise en œuvre du ring (fig.6). Il s'agit d'un axe périphérique circulaire matérialisé aujourd'hui par les cinq rues dénommées nordring, ostring, marienring, südring, westring. Ces nouvelles rues, mais aussi l'espace des anciens glacis ou des ravelins, deviennent des espaces à lotir. Bordant ces artères périphériques, des habitations Art Nouveau (Jugendstil) traduisent l'essor et le dynamisme de la ville. De vastes parcs et jardins publics se déploient au-delà de ces nouvelles artères formant une ceinture verte (fig.6). La ville s'est lancée dans une politique d'offre foncière, 
s'appuyant sur des ressources locales du vignoble, du commerce des matériaux de construction, mais aussi du développement du quartier de la gare à l'est. Il est vrai que la réalisation des grands quartiers militaires de l'armée bavaroise, puis impériale, permet de soutenir la trajectoire urbaine dans un contexte de dilatation de la ville.

11 Avec l'arasement de la forteresse, la ville, tout comme les sites militaires, ne sont plus contraints par le manque d'espace lié à la ceinture de fortifications. Sur le site de l'ancien bastion sud, de vastes quartiers militaires modernes et fonctionnels adaptés à une armée de masse voient le jour. Ils sont destinés à accueillir les $5^{\mathrm{e}}$ et $12^{\mathrm{e}}$ régiments d'artillerie et les $18^{\mathrm{e}}$ et $23^{\mathrm{e}}$ régiments d'infanterie bavarois. La ville doit accueillir un hôpital militaire, des greniers, des logements pour les familles d'officiers, des étatsmajors... Tout un urbanisme militaire façonne alors l'hémisphère sud de la ville. Les liens militaires avec le cœur de ville existent toujours, car certaines casernes, arsenaux ou entrepôts de centre-ville sont toujours en activité. La ville vit de la présence de l'armée dans une symbiose écosystémique de la ville de garnison. Cependant, ces grands quartiers constituent désormais des " capsules ", distinctes de la ville avec une vie interne. Les casernements modernes construits après 1870 répondent aux exigences d'une armée de conscription aux effectifs nombreux. Ils sont plurifonctionnels, ce qui permet à l'armée française d'occuper ceux-ci de 1918 à 1930. Le format de l'armée, sa structure, son matériel, établis sur les mêmes schémas partout en Europe permettent finalement d'occuper les lieux sans adaptation notable de la structure. Le dédoublement de la ville en deux entités civile et militaire facilite cette séparation entre forces occupantes et population dont 35\% est francophone avant 1939. L'organisme urbain, par son métabolisme est dual. C'est la particularité de la ville de garnison.

\section{L'hypermilitarisation d'une petite ville allemande marquée par la présence des F.F.A.}

12 L'armée allemande (1930-1945), puis française (1945-1994) et malgré les destructions de la Seconde Guerre mondiale, réoccupent les espaces militaires. La ville conserve ce statut et cette fonction de ville de garnison, accueille les forces d'occupation, puis des F.F.A. Elle accueille les éléments de la $5^{\mathrm{e}}$ Division blindée et voit passer les régiments (le $13^{\mathrm{e}} \mathrm{RTA}$, le $7^{\mathrm{e}}$ Régiment d'Infanterie, le $8^{\mathrm{e}}$ Régiment d'infanterie, le $2^{\mathrm{e}}$ Régiment d'artillerie, le $5^{\mathrm{e}} \mathrm{RCS}$ de 1978 à 1993, le $44^{\mathrm{e}}$ Régiment de transmissions...). Les importantes infrastructures de casernement et la polyvalence fonctionnelle de ces dernières permettent d'accueillir ces multiples unités et les services qui s'y rattachent. Le domaine immobilier des F.F.A. complète la structure, ajoutant des logements civils afin d'accueillir les familles de militaires, assemblant au noyau des grands quartiers militaires, des services propres aux F.F.A. (poste, tribunal, économat, lycée, école) comme l'a souligné (H. Perrein-Engels, 1994). Durant cette période, Landau accueille une population française de 6000 personnes dont 3000 militaires.

Ce cycle s'achève par une nouvelle phase de démilitarisation qui s'opère comme toujours par la rétrocession à la ville des structures et terrains militaires. Il s'étalonne sur la décennie 1990. Les dernières troupes françaises ont quitté la ville en 1999. Suivant le processus de rétrocession, la ville de Landau a récupéré casernes, cités militaires et terrains. L'ensemble représentait 300 hectares de terrain composés de 36 ensembles immobiliers. La ville devait s'inscrire dans un vaste chantier de 
reconversion/restructuration. Ce processus doit s'établir dans un contexte de crise urbaine, de ville en décroissance. La politique urbaine s'inscrit dans une volonté de préserver l'identité militaire par la conservation et la mise en scène des traces de l'ancienne présence militaire tant vaubanienne que bavaroise ou des F.F.A. Cette démarche de marquage et de patrimonialisation est comparable aux processus décrits par V. Veschambre (2008) ou C. Luxembourg (2013). Elle vise à transformer le non-lieu des friches militaires désormais désertées en hauts-lieux en affirmant et soulignant dans le paysage urbain les traces les plus symboliques pour produire ces marqueurs. Un récit s'organise autour de ces héritages. Ce dernier ne s'inscrit plus à l'échelle nationale voire régionale, mais à l'échelle locale d'une ville de garnison.

\section{Les grandes formes de retraitement des anciens espaces militaires}

\section{A. La valorisation ponctuelle des héritages militaires}

Pour la ville de Landau, la démilitarisation a toujours été un temps permettant de redessiner la ville. Depuis 1945, la question de l'héritage militaire qu'il soit vaubanien ou bavarois, s'inscrit dans une démarche de mise en scène du paysage qui permet à la ville de reconstruire un discours d'une ville marquée par l'histoire de la militarisation et de la mobilité des frontières. Ainsi on peut noter les multiples opérations urbaines qui visent à la conversion de bâtiments militaires au cœur de la ville, mais également en périphérie de cette dernière. Ainsi l'Université de Coblence-Landau voit le jour en 1990, transformant l'Institut de recherche en éducation de Rhénanie-Palatinat (EWH) en pôle universitaire au fonctionnement rhizomique (Coblence, Landau, Mayence). Ce dernier occupe le cœur de l'ancien ouvrage couronné de Landau au nord-ouest de la ville. Le quartier universitaire, uniquement ouvert au sud-est, est bordé d'un écrin matérialisé par les fortifications végétalisées dans un espace de loisirs d'un parc urbain que prolonge le zoo de la ville. Le quartier Chopin (ancien réduit $\mathrm{n}^{\circ} 13$ - fig.7) ou le Hafermagazin (fig.8) ont été mis en scène et reconvertis en lieu de vie avec habitations et commerces (2005-2008). Le Lazarett (ancien hôpital militaire F.F.A. jusqu'en 1996) a été transformé en parc résidentiel associant à la réhabilitation des anciens bâtiments de nouvelles constructions au cœur du parc central.

Toutes ces opérations ponctuelles témoignent du souci de la ville de préserver les traces de son histoire militaire et la mémoire militaire et architecturale des lieux. Cette démarche s'observe également dans le retraitement des grands quartiers militaires établis au sud de la ville. 


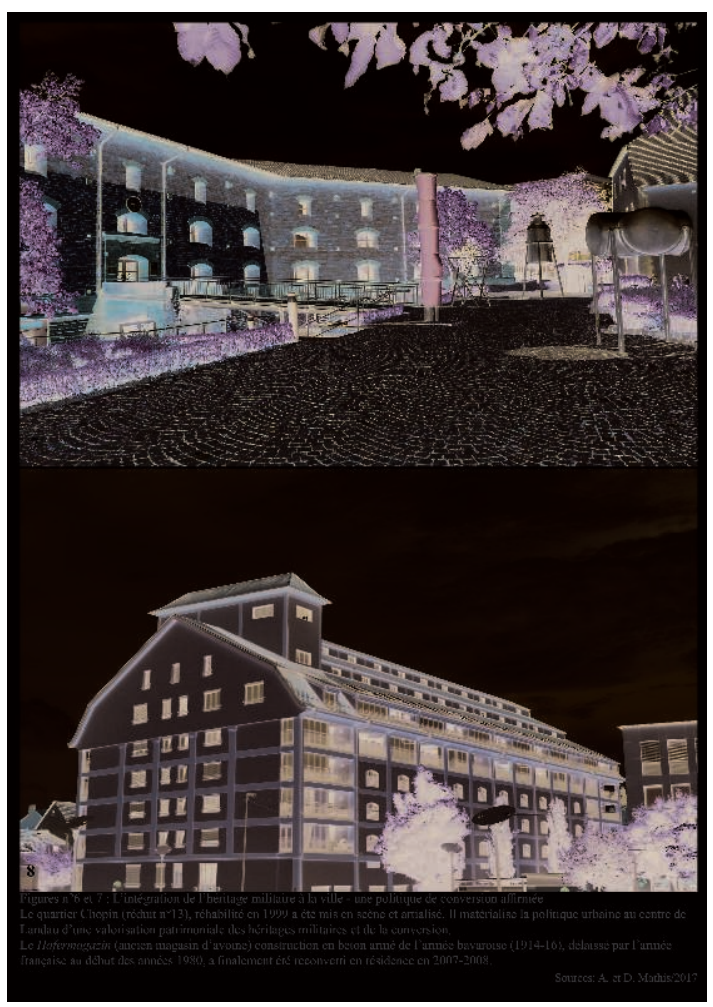

\section{B. La conversion/reconversion des grands quartiers militaires}

La suspension du service national par les armées françaises, dans un contexte géostratégique modifié, conduit à la transformation des territoires de Défense et au retrait progressif des forces stationnées en Allemagne. À Landau, ce départ libère progressivement les implantations militaires du quartier Mangin (1994), du quartier Jeanne d'Arc (1995), du quartier Foch-Estienne et des terrains militaires d'Ebenberg (1999). À ces grands quartiers militaires, il faut ajouter quinze ensembles résidentiels (860 logements), une vingtaine d'îlots techniques et logistiques. C'est un tiers de la surface périphérique de la ville qu'il convient de retraiter en tant que friche militaire. Ce déménagement de l'armée offre à la ville une vaste opportunité foncière pour de grands projets. Les espaces à recycler sont surabondants, mais la prive d'une population nombreuse (environ 6000 habitants - plus de 10\% de la population) et plonge cette dernière dans la quadruple spirale des Stadtschrumpfung Städte ou des shrinking cities (Florentin, Fol, et Roth, 2009 ; Roth, 2011 ; Chatel, 2011) à savoir un choc démographique, socio-économique, financier et urbain. Dans ces conditions, les villes n'ont souvent d'autre choix que d'entamer une contraction ou à une forme de recompactage en raison du surdimensionnement de la ville. Ce processus s'effectue alors par amputation d'une partie importante des espaces urbains démilitarisés. Ce n'est pas l'hypothèse retenue à l'époque à Landau. Les quartiers militaires abandonnés constituent un paysage urbain spécifique de la ville de garnison. 


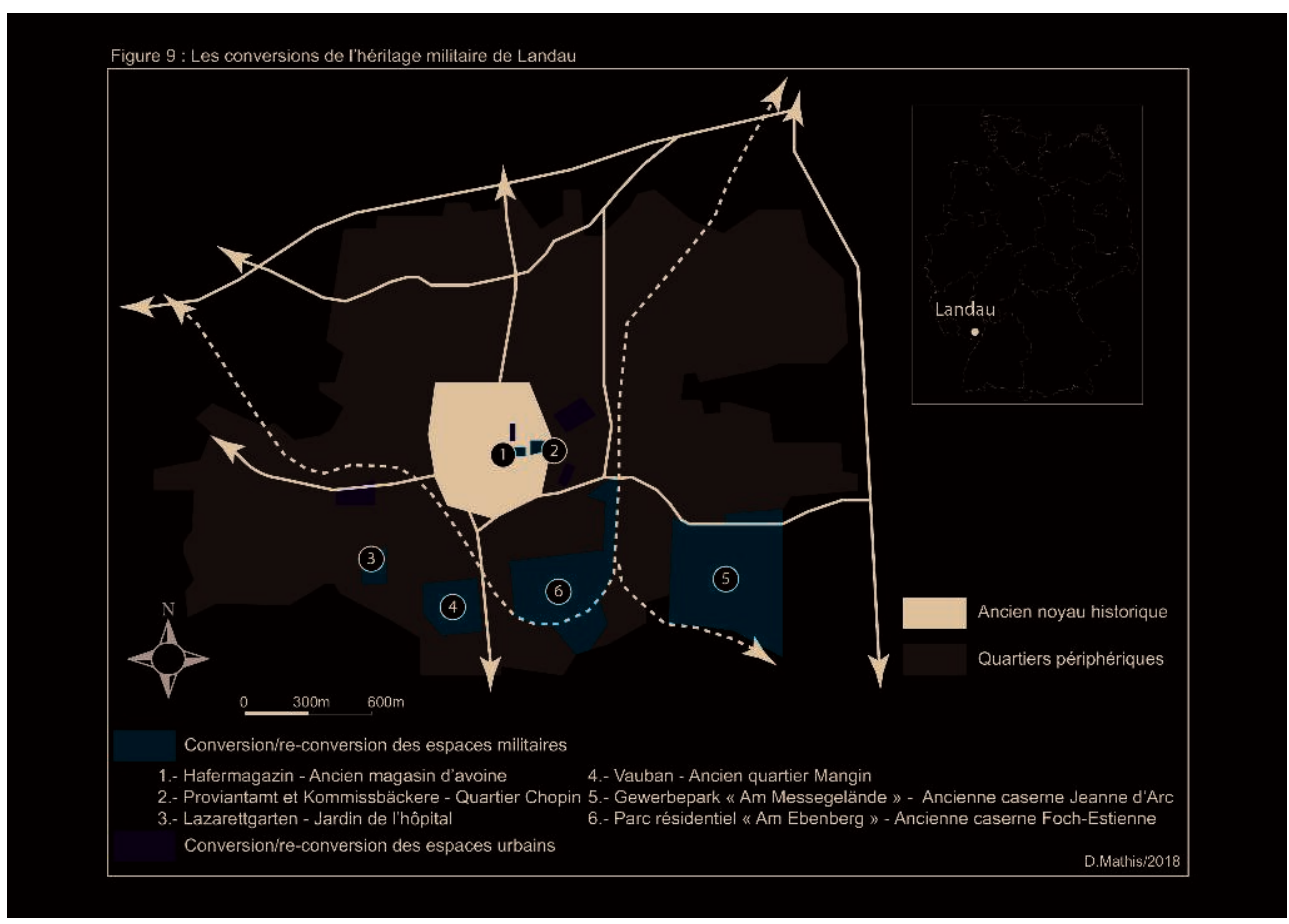

17 La thématique paysagère sert de fil directeur au retraitement sous l'appellation de la "conversion" où l'aménagement urbain doit permettre une reconquête des espaces délaissés pour mettre en application les principes de la charte d'Aalborg. Ainsi, les anciens quartiers militaires vont faire l'objet d'une conversion alternative de la ville afin de produire et de transformer cette dernière en conservant certaines des spécificités propres aux espaces urbains marginalisés afin d'assurer leur reconquête (fig.9). C'est la raison pour laquelle la ville de Landau s'est lancée dans la réalisation innovante d'écoquartiers notamment pour le quartier Mangin, puis Foch-Estienne. Par écoquartier, il faut considérer "un espace bâti nouveau ou reconverti d'une ville, dans ou à proximité d'un centre urbain dense, de l'échelle d'un quartier, ayant pour vocation d'appliquer, de préserver et de développer sur le temps long l'ensemble des principes environnementaux, sociaux et économiques de développement durable qui ont gouverné à sa conception " (Boutaud, 2009). Dans le cas de Landau, la conversion de l'ancien quartier militaire s'inscrit dans une requalification qui tient compte des principes du développement durable et destiné à attirer des habitants dans un quartier désormais ouvert aux civils. La ville s'est lancée dans une politique de «l'offre » dans une démarche de renouvellement urbain, voire de régénération (Chaline, 1999; Mathis et Mathis, 2017). Ce terme nous paraissant plus approprié dans la mesure où les quartiers de casernes restent des espaces fermés et enclavés. Il s'agit d'une « ville militaire » vivant en autarcie tout en influant la vie de la partie civile de la ville.

- Le retraitement expérimental du quartier Mangin : l'écoquartier Vauban (1994-2010)

18 Le quartier militaire Mangin a été le premier espace délaissé, faisant l'objet d'une opération de conversion fonctionnelle. Il faut noter que cette dernière est concomitante à celle de l'écoquartier Vauban à Fribourg en Brisgau en 1994. Intégrer les anciennes casernes dans la ville contraint à recouturer le territoire en ouvrant l'espace du quartier militaire. Le projet défini à partir d'un concours d'idées a permis de construire un projet de "conversion» de l'ancien quartier militaire considéré comme le cœur d'un espace de réhabilitation/rénovation au périmètre plus vaste que l'emprise militaire stricto sensu. La démarche cherche à assurer la préservation des 
constructions militaires considérées pour leur valeur patrimoniale et historique, sans pour autant sanctuariser l'ancien quartier militaire. Le paysage militaire dans sa composition sert d'armature au nouveau quartier de 14,5 hectares. Cette définition d'un périmètre plus vaste que l'ancien quartier doit permettre de recouturer l'espace urbain et d'assurer l'intégration du quartier de casernes dans le tissu urbain. C'est-àdire qu'il s'agit de faire disparaître les enclos de la caserne, d'assurer les transitions entre les espaces.

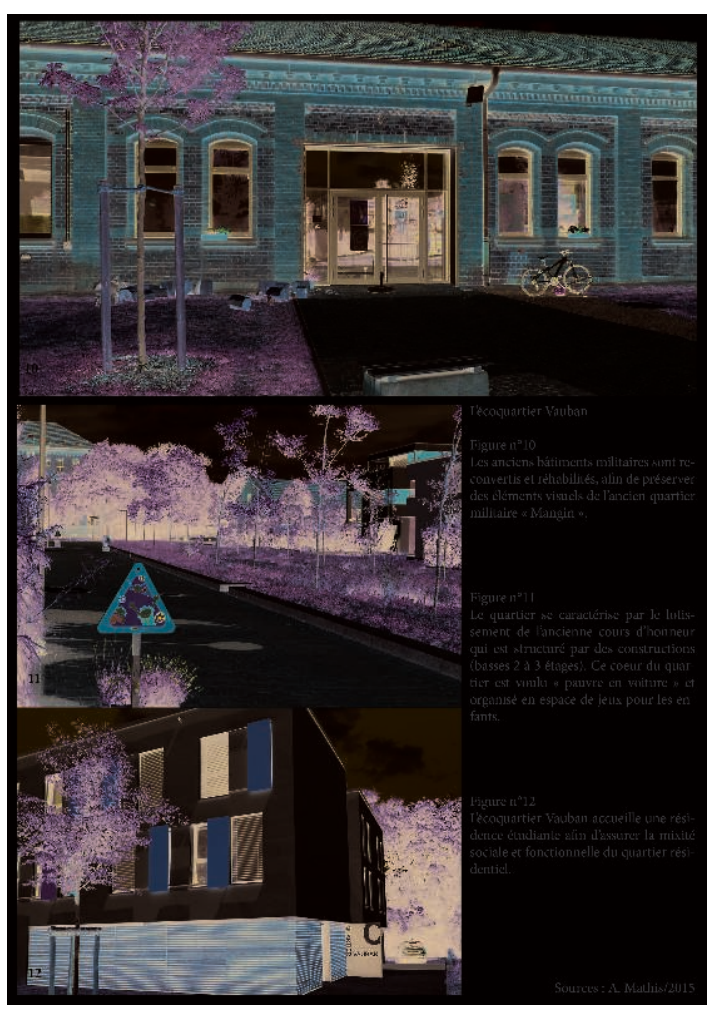

19 Une première étape concerne l'acquisition de terres, la réglementation foncière, la dépollution des sites contaminés, les travaux de démolition, la réalisation de la viabilisation et de l'assainissement, les travaux de conservation-conversion... Les travaux de rénovation ont débuté en 1997 et s'organisent en cinq tranches de travaux qui s'étalonnent de 1994 à 2010. Ils concernent tout autant des formes de maisons individuelles, des résidences collectives dont une cité universitaire, mais aussi des travaux de conservation-conversion des bâtiments de casernes. Au terme de ces phases, 150 maisons et 44 propriétés collectives sous la forme de petits immeubles ont été construites. Seuls 8 bâtiments de l'ancien quartier militaire ont été rénovés et patrimonialisés (fig.10). Ils permettent de conserver une partie du périmètre intérieur de la cour d'honneur qui a été lotie en un îlot central. Ce dernier constitue ainsi une petite unité d'un village urbain, plus qu'un simple quartier résidentiel. Les anciens bâtiments militaires accueillent les services et notamment des écoles. Le quartier accueille environ 500 habitants. La gestion foncière a consacré 2,8 ha aux parcs et espaces ouverts, à la mise en scène de la nature et des jardins. La réalisation de nouvelles formes de logements urbains s'organise suivant le thème de la « vie de jardin " et de la qualité de l'espace ouvert en combinaison avec de nouveaux bâtiments individuels. 2,2 ha pour les zones de circulation et environ 2,3 ha pour l'infrastructure sociale, commerces et services. Le noyau central concentre l'espace de vie et favorise le développement d'un entre-soi. Il permet d'organiser les transports en limitant l'accès à 
l'automobile au noyau central, en assurant à l'ensemble des modes de transport une place équilibrée (fig.11). Enfin le quartier cherche à limiter son impact environnemental, notamment par le développement d'un chauffage collectif utilisant l'énergie géothermique. Au total, cette reconversion Mangin a mobilisé des fonds publics à hauteur de 3,1 millions d'euros pour un volume d'investissement de 16 millions d'euros. Afin d'assurer la transformation identitaire le quartier est renommé Vauban (fig.12).

- Le quartier Jeanne d'Arc : « Am Messegelände »

Délaissé par les F.F.A. en 1995, l'ancien quartier Jeanne d'Arc (11,5 hectares) s'est trouvé au cœur du projet du parc commercial «Am Messegelände» (33 hectares). En 1999-2000, les démolitions sont mises en œuvre, le centre de démarrage a repris un des bâtiments de l'ancienne caserne afin de conserver la trace du quartier militaire et de servir de vitrine au nouveau projet. Là encore la logique reste la même; il s'agit de recouturer partiellement le fil du temps et de l'espace dans un récit traduit spatialement par la conservation d'un patrimoine alibi. Le parc commercial se remplit progressivement à partir de 2001. La gestion du parc industriel et commercial reste souple. La structuration des mobilités s'effectue par des axes à sens unique. L'îlot de l'ancienne caserne inclus dans le nouvel espace industriel relève cependant d'un zonage marqué qui traduit la réhabilitation des espaces urbains compris entre les noyaux de Landau et Queichheim. Ce parc commercial et industriel prend le relais économique du quartier de la gare anticipant la réhabilitation de ce dernier dans la tranche des travaux du quartier Foch-Estienne.

- Du quartier Foch-Estienne au parc résidentiel d'Ebenberg : un quartier qui annonce la ville de demain

21 Le quartier Foch-Estienne était le plus grand quartier militaire (25 ha) et sa réinscription au sein de l'espace urbain constituait un réel défi. En effet, le quartier se prolongeait au sud au-delà de la ligne ferroviaire qui ceinturait les casernements d'un espace de manœuvres et bâtiments techniques se situant à Ebenberg. La couture urbaine se révélait donc difficile à réaliser, le quartier constituant une "capsule " territoriale occupant le sud de la ville. D'autre part, dans le contexte de conversion débuté avec le quartier Mangin, puis le quartier Jeanne d'Arc, il paraissait difficile d'entreprendre une troisième conversion d'une telle ampleur. Cependant dans une démarche et un projet urbain qui s'apparentent à une politique de l'offre, la conversion du quartier militaire Foch-Estienne devait débuter rapidement afin d'éviter un enfrichement qui n'aurait pas permis de s'appuyer sur l'héritage militaire. Il s'agit bien d'une promotion volontariste qui trouve sa transcription dans l'organisation sur l'espace de l'ancienne caserne de l'exposition florale 2014/2015 du Land de Rhénanie Palatinat: le Landesgartenschau. Cependant, comme pour Mangin-Vauban, le projet de requalification est plus global et comprend un périmètre plus vaste d'une trentaine d'hectares. Les travaux démarrent par le nouveau design paysager de la rue Friedrich Ebert et de la Cornichonstrasse, axes de communication reliant le centre-ville et le futur « Wohnpark am Ebenberg ». 


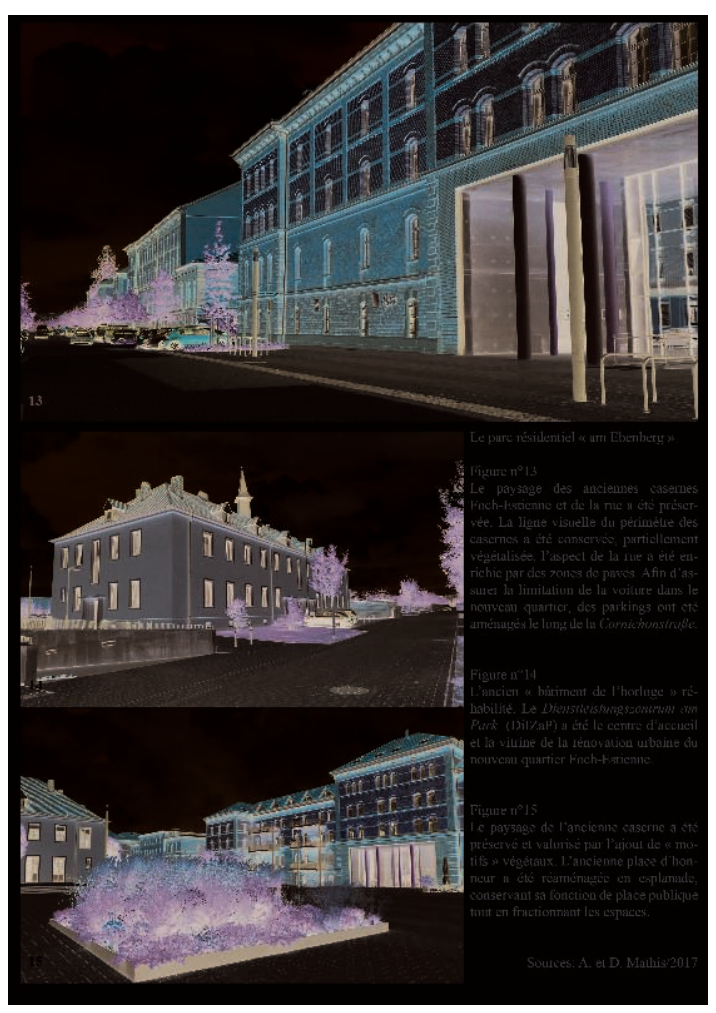

22 L'aspect visuel de la Cornichonstrasse qui borde le quartier militaire conserve l'alignement des grandes casernes maintenant l'enclosure partielle du quartier, tout en aménageant le stationnement périphérique (fig.13). Ce choix permet d'assurer la pérennité d'un paysage hérité de la militarisation. La question de la conservation des identités du quartier militaire passe par la restauration d'une partie des édifices pour leur caractère historique, visuel, esthétique. Cette restauration-réhabilitation est rendue aisée parce que ces vastes bâtiments sont multifonctionnels.

Le Dienstleistungszentrum am Park (DiZaP) symbolise totalement la démarche de mise en scène de l'héritage militaire. En effet, le bâtiment $n^{\circ} 12$ ("Bâtiment de l'Horloge ») de l'ancienne caserne Foch, destiné à servir de lieu de rassemblement et d'instruction a été identifié pour sa valeur patrimoniale (fig.14 et 15). Requalifié, il a servi de vitrine et de centre d'accueil du nouveau quartier dans sa phase de réhabilitation. Il est devenu un immeuble de bureaux, et a été également utilisé comme centre des visiteurs pendant le Landesgartenschau. Le DiZaP accueille le centre de service au Parc. Il est destiné, au terme de la réhabilitation, à être transformé en bureaux. Les grands hangars fonctionnalistes peuvent trouver un réemploi dans le cadre du déploiement de nouveaux services (écoles, bibliothèques de quartiers, jardin d'enfants...). Comme à Vauban, ils peuvent également être affectés en cellules commerciales et de services. Sur Foch-Estienne, ces longs bâtiments techniques sont en cours de réhabilitation ou encore en jachère. Ils fragmentent et délimitent les espaces de l'immense quartier militaire. Ils vont favoriser les îlots commerçants au sein de l'espace de vie et vont servir de lieu de rencontre entre les habitants (fig.16). 


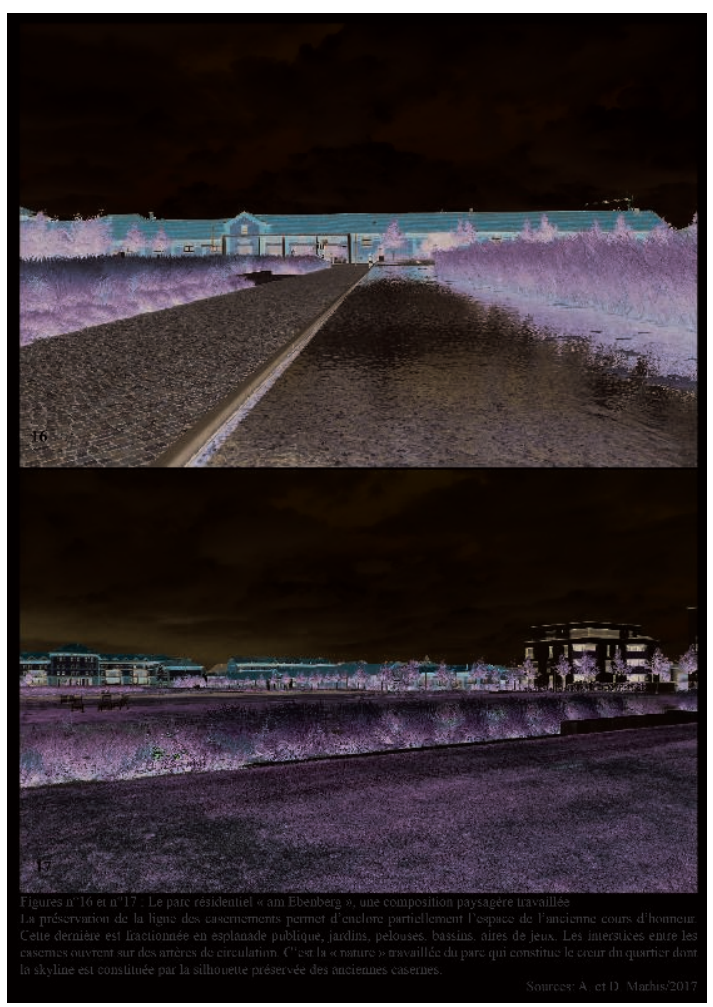

La place d'honneur a été reconvertie en un vaste espace végétalisé, avec jardins, parcs, bassins d'eau, aire de jeu, espace piétonnier, il constitue le cœur paysager du quartier comme jadis la place était au cœur de la vie militaire (fig.17). Cet espace converti a constitué la scène du Landesgartenschau. Cet événement au cœur du projet de retraitement traduit la volonté d'une promotion du paysage comme le souligne E. Bailly (2013) en affirmant trois objectifs :

- image (embellissement et qualité visuelle),

- environnement (nature en ville),

- construction d'un espace public à forte naturalité.

Cependant comme à Mangin/Vauban, la conversion du quartier Foch-Estienne traduit la volonté et l'ambition de conserver la marque de l'armée. Ainsi, conceptuellement, le projet de retraitement élabore un dialogue constant entre l'histoire du quartier militaire : de la présence des F.F.A. jusqu'à l'enfrichement et les étapes des travaux. L'héritage et le devenir du quartier par la réhabilitation sont mis en scène dans une interaction constante entre le paysage urbain et l'environnement. Ce dialogue permet d'annoncer le devenir de l'ancien quartier militaire. Il réinscrit ce vaste chantier de reconstruction, réhabilitation, rénovation dans un cycle passé, présent, futur. La rénovation n'est pas la négation du passé, mais la construction d'un récit à partir du passé militaire. Le style architectural servant de support à la reconquête du quartier a été préservé comme élément identitaire et patrimonial. Il s'agit d'un facteur d'unité paysagère et d'un marqueur qui assurent la rémanence historique, soutiennent la démarche de recyclage et servent d'appui à la régénération de l'espace urbain. Peut-on considérer qu'il s'agit d'une forme de patrimonialisation? Par patrimonialisation, G. Di Méo « revient à poser le principe d'une conservation des biens reçus par héritage (pas tous en fait, une sélection), en vue de leur transmission. Le patrimoine recèle donc la perspective d'une projection dans le futur. Il contient la possibilité d'un avenir qui accroît son caractère d'enjeu stratégique : social, culturel, économique, symbolique et, bien sûr, territorial. » (2008). Ainsi, 
dans la démarche, la patrimonialisation n'est pas la stérilisation d'un territoire urbain à rénover, mais bien une projection de ce dernier dans une démarche où la sélection des édifices les mieux adaptés au recyclage et/ou les traces ou les agencements les plus à même de soutenir un récit urbain permettent de construire la démarche de transition (fig.18 et 19). Le paysage soutient le récit et sert la démarche opératoire (Sgard, 2008 ; Sgard, 2010 ; Ginet et et Wiesztort, 2013). En cela, la valorisation et l'affirmation de l'héritage militaire comme élément culturel d'un cadre de vie sert de vecteur à la fabrique de la ville. Ainsi cela dépasse le constat de B. Grésillon qui affirmait que « ${ }^{\circ}$ lorsque la politique culturelle est associée à une vision d'ensemble qui combine politique de réhabilitation de logements et politique économique visant à rendre attractif un territoire autrefois déprécié, elle peut être efficace, au moins en termes de changement d'image » (2011).

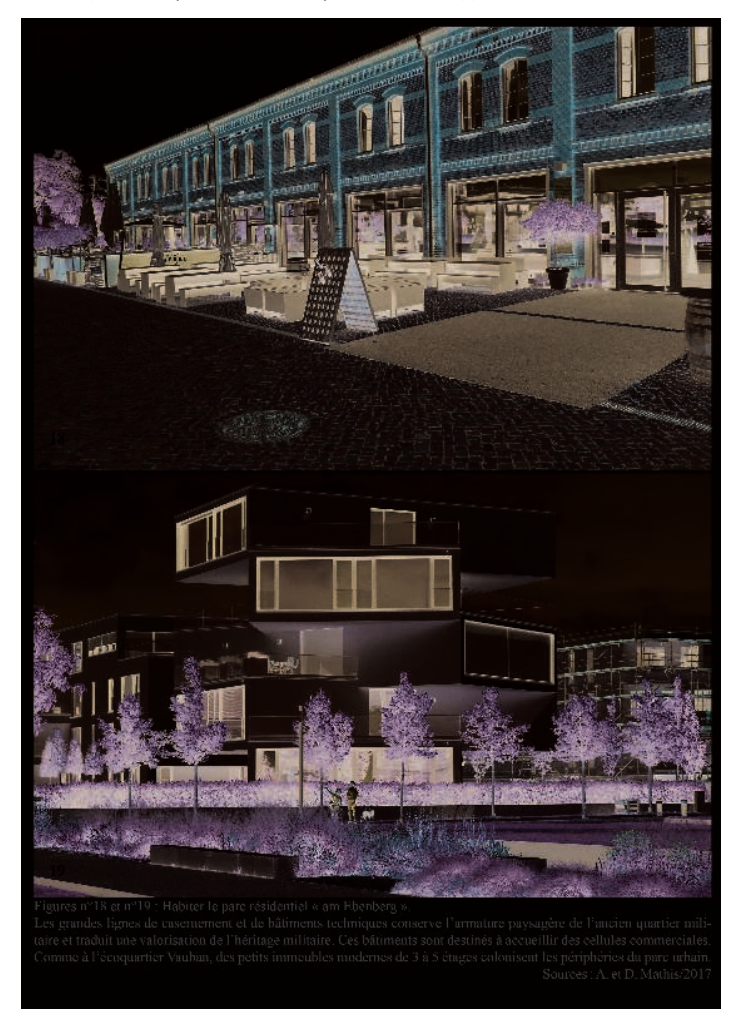

Le parc résidentiel am Ebenberg comme Vauban est un écoquartier par la valorisation des héritages militaires, ses démarches environnementales, de mixités sociales et fonctionnelles, de vivre ensemble, d'entre-soi et de gentrification mis en œuvre dans le projet urbain d'une ville durable. La qualité environnementale d'une nature travaillée traduite visuellement par l'immense jardin est au cœur de la conversion urbaine. La structuration des mobilités a été importante sans prendre une place aussi considérable que dans la reconversion de l'écoquartier Vauban à Fribourg en Brisgau. La valorisation de l'héritage militaire est, dans l'exemple de Landau, le principal discours qui soutient la conversion en vue de régénérer les espaces urbains.

\section{Une forme urbaine conservée, un tissu urbain régénéré.}

27 En changeant d'échelle, il apparaît que la ville de Landau a profondément recomposé son espace urbain péricentral au sud de son ancien centre historique. La réinscription 
des casernes au sein de la trame urbaine a permis de redynamiser également les périphéries, partiellement déclassées des périmètres militaires, et d'étendre la rénovation urbaine au quartier sud de la gare. Ainsi, dans un contexte de redéfinition de son espace urbain, l'ancien quartier militaire délaissé est une opportunité pour concevoir un projet global dans une démarche de planification urbaine entreprise par la ville. La rénovation urbaine et la préservation de l'héritage de l'urbanisme militaire sont privilégiées dans cette planification urbaine. Pour la ville, la question militaire permet de construire un récit et se traduit par un empilement des héritages dont le cœur reste vaubanien. Bien qu'inachevée, la réhabilitation péricentrique de Landau a eu un effet positif concernant la dynamique urbaine. Ainsi, le noyau urbain de Landau a connu une croissance de sa population de 1\% par an sur la période entre 2011 et 2015. Cette politique d'offre a permis au cœur urbain d'enregistrer une croissance plus marquée que celle du reste de l'agglomération, limitant l'étalement urbain.

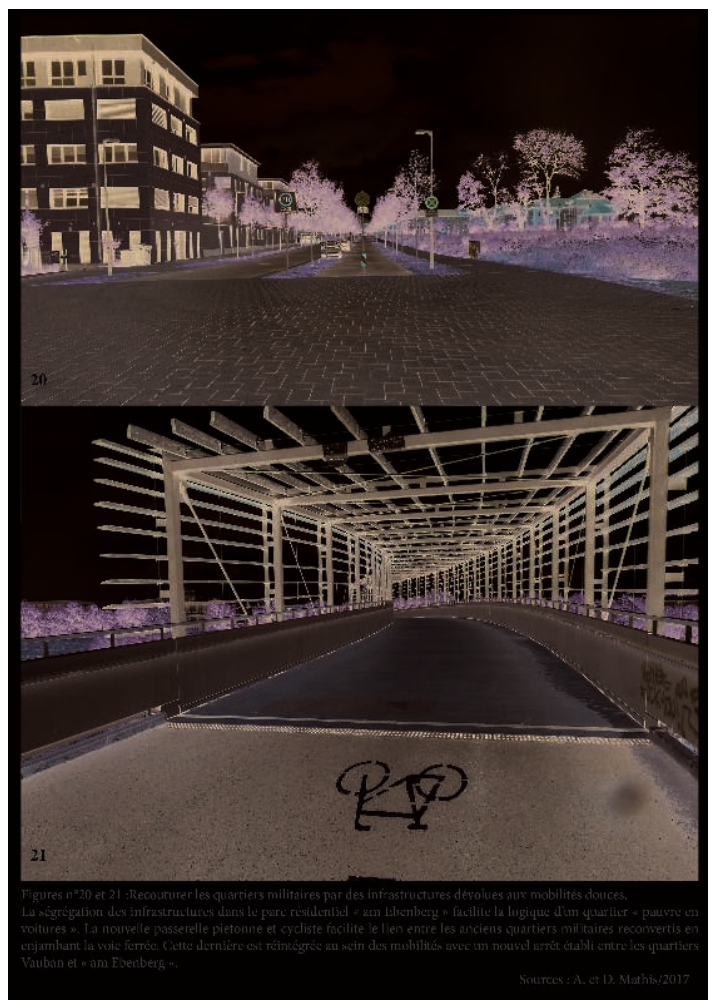

Ville pionnière dans le retraitement des espaces urbains délaissés, la ville de Landau applique des méthodes de planification et de standardisation de la ville durable. Depuis l'écoquartier Vauban, puis le nouveau quartier am Ebenberg, les lieux de la ville sont retravaillés en utilisant la logique du fractionnement des espaces de circulation (piéton, vélo, voiture) (fig. 20 et 21 ). Le réseau de pistes cyclables du quartier a été relié au réseau du centre-ville avec des prolongements vers Ebenberg et Queichheim, notamment par une grande passerelle qui franchit la ligne de chemin de fer. Les places de stationnement pour les voitures sont pour la plupart situées à l'extérieur de l'espace public, notamment le long de la Cornichonstrasse, à l'entrée du quartier, mais aussi dans des garages souterrains. L'objectif affiché de faire des rues du quartier des espaces de vie libérés des voitures annonce un quartier "pauvre en voitures ». Afin d'éviter l'engorgement du quartier la «nouvelle entrée»sur Cornichonstrasse reste une ouverture partielle. Le quartier privilégie les transports doux. À l'échelle de l'ensemble des quartiers sud, les mobilités sont organisées également à partir de la nouvelle gare 
de Landau Süd reliant le parc résidentiel am Ebenberg et plus globalement les 5500 habitants de la ville sud, assurant ainsi une meilleure connexion entre lieu de travail et de résidence. Ce nouvel arrêt de chemin de fer sur l'axe Landau-Pirmasens répond aux enjeux de reconquête des anciens quartiers militaires. Cette gare annexe est destinée à assurer le rôle de plateforme intermodale, intégrant cette dernière au projet de Landesgartenschau.

Les héritages de la militarisation sont aujourd'hui réintégrés à l'espace urbain comme un marqueur spécifique de la ville. La conversion des nouveaux quartiers permet de mettre en chantier la redynamisation de l'ensemble de l'espace sud-est de la ville. Au cœur du projet urbain, le retraitement des casernes est finalement extrêmement normé et standardisé avec :

- le verdissement et la mise en chantier de jardins paysagers,

- la réorganisation des mobilités et les aménagements d'axes spécifiques aux modes de transport,

- la préservation patrimoniale des bâtiments, la conversion fonctionnelle et la mise aux normes environnementales,

- la gestion collective et la mixité sociale, favorables à développer un entre-soi,

- la gestion énergétique des quartiers (géothermie),

- la mise en œuvre des espaces publics.

Comme le souligne B. Boutaud (2009), «les écoquartiers dans l'ensemble des pays d'Europe du nord se sont mués du modèle de «quartiers prototypes » en "quartiers types ». Cependant, la vision techniciste et normative de l'écoquartier à Landau ne doit pas faire oublier que cette production relève également de pratiques habitantes participatives et de formes d'écocitoyenneté. En cela, il ne s'agit pas d'un modèle utopique dupliqué, mais bien d'un projet collectif.

31 L'action volontariste de la municipalité effectuée sur le métabolisme urbain de Landau répond aux questions de villes décroissantes, mais aussi aux enjeux de la ville durable. Conserver une approche géohistorique et paysagère à cette étude a permis de mettre en avant la spécificité de la ville démilitarisée par l'affirmation de marqueurs mémoriels de ses anciennes fonctions militaires. Ces marqueurs, qu'ils soient d'époques modernes ou contemporaines accompagnent la mise en scène du paysage, un paysage palimpseste qui n'est plus militarisé, mais dont on a conservé certaines traces afin de limiter la banalisation et l'uniformisation de la ville durable dont les normes conduisent finalement à une standardisation. Ainsi, le paysage urbain post-militaire obtenu par la conversion accompagne une transition progressive d'un état à un autre. Il est paradoxal de considérer qu'un espace militaire normé et standardisé par les besoins et les contraintes fonctionnelles soit aujourd'hui le support d'un récit destiné à limiter l'uniformisation de la ville. Il met en lumière des formes architecturales et structurelles du quartier afin de construire et restaurer un métabolisme économique, social et environnemental d'un espace à l'origine uniquement destiné et construit par l'activité militaire. Au-delà de la question de la transition militaire, Landau s'inscrit dans une logique de planification urbaine et dans un véritable projet urbain: "landau-bautzukunft » afin de réaliser d'ici 2030, 1200 unités résidentielles nouvelles dont 900 pour le quartier am Ebenberg . 


\section{Conclusion}

Cette approche géohistorique des dynamiques urbaines de la ville de Landau a permis de souligner les formes de transitions entre les formes urbaines héritées des territoires de défense et les paysages urbains. Ces transformations opérées fin XIX siècle pour une ville forteresse transformée en ville de garnison moderne, s'opèrent aujourd'hui dans une transition vers la ville post-militaire. Le paysage militaire hérité est déconnecté de sa fonction d'origine pourtant il est conservé comme marqueur identitaire. Cette transition des formes de la ville s'est opérée rapidement et finalement illustre l'importance du projet urbain dans les processus de transition et de régénération. De l'expérimentation Vauban au projet résidentiel am Ebenberg, cet exemple de Landau souligne l'implication d'acteurs multiples dans une démarche volontariste et globale. À l'heure où de nombreuses villes doivent encore gérer leurs héritages militaires comme un long "chemin de croix» (Mathis, 2016, Artioli, 2016a et 2016b), les conversions s'inscrivent dans un récit mémoriel, qui valorise un héritage tout en entamant un vaste processus de ré-urbanisation. En préservant et en s'appropriant son héritage militaire la ville inscrit celui-ci dans son patrimoine urbain et construit ce-dernier dans son paysage culturel.

33 La politique de la ville a cherché à enrayer la décroissance urbaine liée à la perte de la fonction militaire et d'une population civile et militaire en pratiquant une politique destinée à produire de l'urbain. Il faut reconnaître que la ville s'est donnée les moyens d'assurer cette transition de la ville de garnison à la ville post-militaire, comme elle a su gérer la transition entre la cité guerrière et la ville de garnison. Elle a profité de l'opportunité du déclassement des infrastructures militaires pour redessiner sa trame urbaine et régénérer des pans entiers de la ville.

34 Au-delà de la question de transition des anciennes villes de garnison des F.F.A., Landau illustre les processus actuels globaux que révèlent les transitions urbaines des villes de garnison. Elle représente une trajectoire où la démilitarisation et la mutation fonctionnelle de la ville sont considérées comme une opportunité de régénération urbaine plutôt qu'une simple opportunité foncière pour inscrire des activités dévoreuses d'espace » dans une opération de rénovation faisant table rase des identités et des spécificités. La lecture de la ville rénovée, reconstruite sur elle-même s'organise ici dans une lecture patrimoniale de valorisation des héritages.

Cette étude nous permet de souligner les continuités conceptuelles dans la production de la ville post-militaire. La ville a su transformer le départ de l'armée en un atout, sans entrer dans des processus de décroissance, de recompactage ou de démarches opportunistes destinées à occuper l'espace friche. Le départ de l'armée a été géré en planifiant un projet urbain global et à l'échelle de la ville et de l'aire urbaine. Ce retraitement planifié est un exemple à transposer à l'échelle européenne pour régénérer les villes de garnisons. Il montre que la décroissance urbaine n'est pas une fatalité et qu'un projet urbain valorisant les héritages permet d'assurer la transition et la régénération urbaine. L'identité liée à la militarisation est partiellement préservée, la reconversion s'est opérée dans une logique de valorisation d'une partie de l'héritage architectural militaire. Elle prolonge en cela la volonté de revaloriser le patrimoine vaubanien au sein de la ville-centre avec la reconstruction d'éléments fortifiées telles les lunettes $n^{\circ} 38$ et $41^{8}$ ou la mise en œuvre d'un circuit touristique «Vauban ». 

une logique d'abandon et de délaissement d'espaces spécifiques voués à l'armée. Pourtant elle permet la construction d'un imaginaire et d'une identité, d'une fierté de la ville de garnison dans ses multiples héritages perçus non pas à l'échelle de l'affrontement des nations mais d'une vie, d'un habiter construit dans une relation entre la garnison, ses familles, ses habitants. La conservation d'une part de cet héritage est naturelle car pour les habitants il a une valeur évidente. Cependant cette valorisation dépasse le cadre d'un patrimoine muséifié mais cherche à transmettre un patrimoine vivant, paysage intégré à la ville, soutenant le récit des transitions urbaines. La volonté actuelle de ré-exhumer les vestiges des anciens remparts et fortifications à Landau mais aussi à Saarlouis traduit l'affirmation de marqueurs devenant des géosymboles des anciennes cités guerrières et villes de garnison en Allemagne.

La question de la démilitarisation touche finalement de multiples villes en Europe notamment dans les grandes régions à vocation militaire ou les identités ont été marquées par la présence de l'armée (région Grand-Est, Frioul-Vénétie Julienne, Allemagne de l'ouest). Pour les zones de déploiements des F.F.A. 73 villes de garnison et l'écoquartier Vauban à Fribourg en Brisgau a masqué un processus de transition qui n'est pas achevé. Par sa démarche Landau montre par son choix de «construire l'avenir » en conservant les marques et les identités de l'armée une trajectoire de conversion remarquable.

\section{BIBLIOGRAPHIE}

Artioli Fr., 2016a, «Les politiques des fonciers publics. La reconversion des sites militaires dans les villes françaises et italiennes entre réformes des armées, contraintes budgétaires et aménagements urbains ", Métropoles [En ligne], 18 | 2016, mis en ligne le 15 juin 2016, consulté le 25 juin 2018. URL : http://journals.openedition.org/metropoles/5244

Artioli Fr., 2016b, « Restructurations du centre et conflits des périphéries. L'échec des projets urbains face au retrait des armées en Italie », Revue française de science politique, 2016/2 (Vol. 66), p. 229-250. DOI : 10.3917/rfsp.662.0229. URL : https://www.cairn.info/revue-francaise-de-sciencepolitique-2016-2-page-229.htm

Bailly E., 2013, « Des espaces publics aux espaces paysagers de la ville durable », Articulo - Journal of Urban Research [Online], Special issue 4 | 2013. URL : http://articulo.revues.org/2233

Boutaud B., 2009, «Quartier durable ou éco-quartier? », Cybergeo : European Journal of Geography [En ligne], Débats, Quartier durable ou éco-quartier?, mis en ligne le 24 septembre 2009, consulté le 16 octobre 2017. URL : http://cybergeo.revues.org/22583

chaline C., 1999, La régénération urbaine, Que sais-je ? Paris, Presses Universitaires de France, $127 \mathrm{p}$.

Cauchi-Duval N., Béal V. et Rousseau M., « La décroissance urbaine en France : des villes sans politique », Espace populations sociétés [En ligne], 2015/3-2016/1|2016, mis en ligne le 20 mars 2016, consulté le 01 août 2017. URL : http://eps.revues.org/6112 
Chatel C., 2012, « Une mesure du déclin démographique des villes allemandes de 1820 à 2010 ", Géocarrefour [En ligne], Vol. 86/2 | 2011, mis en ligne le 05 mars 2012, consulté le 01 août 2017. URL : http://geocarrefour.revues.org/8295

Di Méo G., 2008, Processus de patrimonialisation et construction des territoires. Patrimoine et industrie en Poitou-Charentes : connaître pour valoriser, Sept 2007, Poitiers-Châtellerault, France, Geste éditions, pp.87-109.

Elissalde B., 2000, « Géographie, temps et changement spatial ». In : Espace géographique, tome 29, n³, 2000. pp. 224-236. www.persee.fr/doc/spgeo_0046-2497_2000_num3_2008

Engels-Perrein H., 1997, La présence militaire française en Allemagne de 1945 à 1993, Mosella, Tome XXII, numéro spécial annuel, 302p.

Florentin D., Fol S. et Roth H, 2009, « La "Stadtschrumpfung" ou "rétrécissement urbain" en Allemagne : un champ de recherche émergent », Cybergeo : European Journal of Geography [Online], Space, Society,Territory, document 445, Online since 26 March 2009, connection on 16 October 2017. URL : http://journals.openedition.org/cybergeo/22123

George P. 1967, « Le temps géographique », Cahiers de géographie du Québec, 1124, (1967), pp. 469-477.

Ginet P. et Wiesztort L., 2013, « La place de la mémoire dans les aménagements territoriaux, un enjeu géopolitique. ", Revue Géographique de l'Est [En ligne], vol. 53 / 3-4 | 2013, mis en ligne le 02 juillet 2014, consulté le 22 octobre 2017. URL : http://journals.openedition.org/rge/5059

Grésillon B, 2011, « La culture comme alternative au déclin : mythe ou réalité ? ", Géocarrefour [En ligne], Vol. 86/2 | 2011. URL : http://geocarrefour.revues.org/8305

JALABERT L., 2011, « La politique territoriale française sur la rive gauche du Rhin (1679-1697) : des « réunions » à la Province de la Sarre », Revue historique, 2011/1 (n 657), p. 61-91. URL : http://www.cairn.info/revue-historique-2011-1-page-61.htm

Luxembourg C., 2013, Patrimonialiser, revitaliser, habiter l'industrie en ville : une question politique et sociale vivante plus qu'une simple question de renouveau urbain, Revue Géographique de l'Est, vol. 53 / 3-4 | 2013, mis en ligne le 3012 2013, consulté le 2508 2014, URL : http:// rge.revues.org/5105

Mathis D., 2011, « «Quand l'armée s'en va ». Approche géohistorique des restructurations des espaces militarisés : l'exemple du sud mosellan », Revue Géographique de l'Est [En ligne], vol. 51 / 1-2 | 2011. URL : http://rge.revues.org/3249

Mathis D., 2016a, «La démilitarisation des villes : la difficile résorption de marges urbaines » in Wackermann G., (dir.), La France des marges, Paris, Ellipses, 528 p., p.509-514.

Mathis D., 2016b, « Les hydrosystèmes militaires défensifs de Basse-Alsace (XVII ${ }^{\mathrm{e}-X V I I I^{\mathrm{e}}}$ siècles) ", Revue de Géographie Historique, ${ }^{\circ} 8$, Géographie historique et questions militaires (1), http:// rgh.univ-lorraine.fr/articles/view/70/

Les_hydrosystemes_militaires_defensifs_de_Basse_Alsace_XVIIe_XVIIIe_siecles

Mathis D. et Mathis A., 2015, « Démilitarisation et reconversion de l'héritage militaire », in Projets de paysage [En ligne], dossier thématique $\mathrm{n}^{\circ} 11$, , http://www.projetsdepaysage.fr/ demilitarisation_et_reconversion_de_l_heritage_militaire

Mathis D. et Mathis A. 2017, «Régénération de la conurbation sidérurgique du Val-de-Fensch (Moselle-France) », Territoire en mouvement Revue de géographie et aménagement [En ligne], 33 | 2017. URL : http://tem.revues.org/4039 
ROCOLLE P., 2002, « La fin des cités guerrières au XIX ${ }^{\mathrm{e}}$ siècle dans la France du Nord. Essai de synthèse », Revue du Nord, 2002/1 (n 344), p. 51-67. URL : https://www.cairn.info/revue-dunord-2002-1-page-51.htm

Roth H., 2011, « Les « villes rétrécissantes » en Allemagne », Géocarrefour [En ligne], Vol. 86/2 | 2011, mis en ligne le 05 mars 2012, consulté le 28 juillet 2017. URL : http:// geocarrefour.revues.org/8294

Sgard A., 2008, « Entre rétrospective et prospective », EspacesTemps.net, Travaux, 26.09.2008, https://www.espacestemps.net/articles/entre-retrospective-et-prospective/

Sgard A., 2010, « Le paysage dans l'action publique : du patrimoine au bien commun », Développement durable et territoires [En ligne], Vol. 1, $n^{\circ} 2 \mid$ Septembre 2010, mis en ligne le 23 septembre 2010, consulté le 01 août 2017. URL : http:// developpementdurable.revues.org/8565

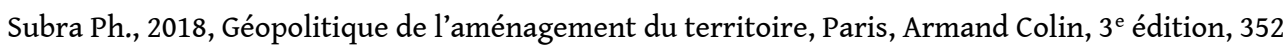
p.

Veschambre, V., 2008, Traces et Mémoires urbaines. Enjeux sociaux de la patrimonialisation et de la démolition, Rennes, Presses universitaires de Rennes, coll. «Géographie sociale », 315 p.

VON HIRSCHHAUSEN B., 2017, « De l'intérêt heuristique du concept de «fantôme géographique » pour penser les régionalisations culturelles », L'Espace géographique, 2017/2 (Tome 46), p. 106-125. URL : https://www.cairn.info/revue-espace-geographique-2017-2-page-106.htm

Von Hirschhausen B., 2017, « Leçon des frontières fantômes : les traces du passé nous viennent (aussi) du futur ", L'Espace géographique, 2017/2 (Tome 46), p. 97-105. URL : https:// www.cairn.info/revue-espace-geographique-2017-2-page-97.htm

\section{NOTES}

1. Livre blanc sur la Défense, 1994, p 4, consulté le 01/08/2017 http:// www.livreblancdefenseetsecurite.gouv.fr/archives-Livre-blanc-1994.html.

2. Les frontières fantômes désignent les traces laissées dans l'espace contemporain par d'anciennes frontières historiques. Ces anciennes constructions du passé demeurent prégnantes ou réapparaître dans les territoires notamment dans les identités ou encore les réseaux d'infrastructures qui peuvent être remobilisés (VON HIRSCHHAUSEN, 2017 et 2017).

3. Le concept de « ville de demain » ou « ville du futur »- prolonge la question de la ville durable. Il est à la fois opératoire et prospectif c'est-à-dire que la ville de demain résulte d'un certain nombre de choix d'aménagement qui sont faits aujourd'hui. Ce concept est exprimé au sein du rapport sénatorial, Sueur J-P. (s. d.), Villes $d u$ futur, futur des villes. Quel avenir pour les villes du monde?, rapport d'information fait au nom de la délégation sénatoriale à la prospective sur les villes du futur, Paris, Sénat, 2011, http://www.senat.fr/rap/r10-594-1/r10-594-11.pdf. Elle revêt plusieurs aspects développés à titre expérimental en France dans les projets d'écocité. À Landau, le projet urbain de conversion des friches militaires s'inscrit dans la démarche prospective Landau construit l'avenir - « Landau-baut-zukunft » à l'horizon 2030.

4. En 2016.

5. Kreis-freie Stadt est une ville qui n'est pas intégrée au sein d'un Kreis - arrondissement. Il s'agit donc d'une ville «libre » qui forme son propre arrondissement. Ainsi autour de Landau gravitent les quartiers d'Arzheim, de Dammheim, de Godramstein, de Mörlheim, de Mörzheim, de Nußdorf, de Queichheim et de Wollmesheim. 
6. Caserne d'artillerie (1692), Caserne Blanche (1700), Reiterkaserne (1710), Casernes rouges 1756 à 1759 , Caserne du fort.

7. Après étude prospective, la ville de Landau devrait augmenter sa population de $5,2 \%$ entre 2014 et 2030 pour atteindre environ 47400 habitants.

8. Ouvrage avancé ou détaché de l'architecture bastionnée.

\section{RÉSUMÉS}

Les villes de garnison sont des villes contraintes et organisées par l'armée. La mutation structurelle de l'espace urbain marquée par les besoins des armées inscrit ses transformations sur un temps long. La ville de Landau in der Pfalz en Rhénanie-Palatinat illustre parfaitement les étapes des processus de militarisation de la ville forteresse dessinée par Vauban, devenue par la suite une importante ville de garnison et finalement aujourd'hui une ville post-militaire. Les transformations des fonctions militaires, puis la perte de celles-ci, remettent en cause le système militaro-urbain et posent la question du devenir des infrastructures militaires avec le risque d'entrer dans un schéma de villes décroissantes et rétrécissantes, en sacrifiant ses héritages militaires. À Landau, la valorisation du patrimoine militaire, ancien ou contemporain, constitue une opportunité et une ressource permettant de restructurer la ville, et finalement de construire un modèle de transition urbaine de la ville post-militaire.

The garrison towns are forced cities organized by the army. The structural change of urban space marked by the needs of the armed forces are its transformations over a long time. The city of Landau in der Pfalz, in Rhineland Palatinate, is a perfect example and steps of the process of militarization of the city fortress designed by Vauban. Later Landau became an important garrison town and finally a post-military city today. Transformations and the loss of military functions calls the question of the military-urban system and the future of the military infrastructure with the risk of getting into a pattern of shrinking cities, by sacrificing its military legacies. The military heritage is an opportunity and a resource to restructure the city, and eventually to built a post-military-city model of transition.

Garnisonsstädte sind Städte, die einseitig von der Armee geprägt und organisiert werden. Der strukturelle Wandel des städtischen Raums, der sich (primär) an den Bedürfnissen der Armeen orientiert, führt zu lange nachwirkenden Veränderungen. In Landau (Rheinland-Pfalz) spiegeln sich deutlich die Schritte des Militarisierungsprozesses der von Vauban entworfenen Festungsstadt, die später eine wichtige Garnisonsstadt und schließlich heute eine „PostMilitärstadt" geworden ist. Der Wandel der militärischen Funktionen bis schließlich zu ihrem Verlust stellen das System einer Militärstadt in Frage, machen aber auch die Zukunft der militärischen Infrastruktur selbst ungewiss, denn mit der Preisgabe des Militärerbes wächst das Risiko, in die Abwärtsspirale der schrumpfenden Städte zu geraten. In Landau stellt die Inwertsetzung des ehemaligen und heutigen militärischen Erbes eine Chance und eine Ressource dar, die Stadt zu restrukturieren und schließlich ein städtisches Modell für den Übergang zur „Post-Militärstadt“ zu entwickeln. 
INDEX

Mots-clés : Rhénanie-Palatinat, ville forteresse, ville de garnison, démilitarisation, reconversion, transition, shrinking-city

Keywords : Rhineland-Palatinate, Garrison towns, demilitarization, conversion, transition, shrinking-city

Schlüsselwörter : Rheinland-Pfalz, Festungstadt, Garnisonstadt, Entmilitarisierung, Konvertierung, Übergang, schrumpfende Städte

\section{AUTEURS}

\section{DENIS MATHIS}

Maître de conférences en géographie, Université de Lorraine Laboratoire d'Observation des TERRitoires (LOTERR)

23, boulevard Albert 1er - BP 33-7 - 54015 NANCY CEDEX

Tél : 03.54.50.51.27

denis.mathis@univ-lorraine.fr

\section{ANNE MATHIS}

Chercheuse associée - Université de Lorraine Laboratoire d'Observation des TERRitoires (LOTERR) 23, boulevard Albert 1er - BP 33-97 - 54015 NANCY CEDEX Tél : 03.54.50.51.27

Mail : Mathisanne@aol.com 